\title{
OSCILLATION CRITERIA FOR SECOND ORDER NONLINEAR NEUTRAL DYNAMIC EQUATIONS ON TIME SCALES
}

\author{
E. THANDAPANI AND V. PIRAMANANTHAM
}

\begin{abstract}
In this paper, the authors established some new oscillation criteria for the second order nonlinear neutral delay dynamic equations on time scales. Examples illustrating the main results are given.
\end{abstract}

\section{Introduction}

In recent years, there has been much research activity concerning the oscillation of solutions of second order dynamic equations on time scales, see for example [1-12, 17, 19] and the references contained therein. However, there are few results dealing with the oscillation of solutions of neutral delay dynamic equations on time scales, see for example [15, 16, 18]. Motivated by this observation, in this paper we are concerned with the second order nonlinear neutral delay dynamic equation on time scales

$$
\left(r(t)\left((y(t)+p(t) y(t-\tau))^{\Delta}\right)^{\gamma}\right)^{\Delta}+q(t) y^{\beta}(t-\delta)=0, \quad t \in \mathbb{T},
$$

where $\mathbb{T}$ is a time scale.

Throughout this paper we assume the following conditions without further mention:

$\left(H_{1}\right) \gamma \geq 1$, and $\beta>0$ are quotients of odd positive integers;

$\left(H_{2}\right) \tau, \delta$ are fixed nonnegative constants such that the delay functions $\tau(t)=t-\tau<t$ and $\delta(t)=t-\delta<t$ satisfy $\tau(t): \mathbb{T} \rightarrow \mathbb{T}$ and $\delta(t): \mathbb{T} \rightarrow \mathbb{T}$ for all $t \in \mathbb{T} ;$

$\left(H_{3}\right) \quad q(t)$ and $r(t)$ are real valued rd-continuous positive functions defined on $\mathbb{T}$;

$\left(H_{4}\right) \quad p(t)$ is a positive and rd-continuous function on $\mathbb{T}$ such that $0 \leq p(t)<1$.

By a solution of equation (1.1), we mean a nontrivial real-valued function which has the properties $y(t)+p(t) y(t-\tau) \in C_{r d}^{\prime}\left[t_{y}, \infty\right)$ and $r(t)\left([y(t)+p(t) y(t-\tau)]^{\Delta}\right)^{\gamma} \in C_{r d}^{\prime}\left[t_{y}, \infty\right), t_{y} \geq$ $t_{0}$ and satisfying equation (1.1) for all $t \geq t_{y}$. A solution $y(t)$ of equation (1.1) is said to be

Corresponding author: .

2010 Mathematics Subject Classification. Primary 34K11; Secondary 39A10, 39A99.

Key words and phrases. Oscillation, second order, neutral dynamic equations, time scales. 
oscillatory if it is neither eventually positive nor eventually negative, that is, if for every $b>a$ there exists $t>b$ such that $y(t)=0$ or $y(t) y(\sigma(t))<0$; Otherwise it is called nonoscillatory.

Since we are interested in oscillatory behavior of solutions, we will suppose that the time scale $\mathbb{T}$ under considerations is not bounded above and therefore the time scale is assumed in the form $\left[t_{0}, \infty\right)_{\mathbb{T}}=\left[t_{0}, \infty\right) \cap \mathbb{T}$.

In this paper we obtain the oscillation criteria for equation (1.1) subject to the following two conditions:

$$
\int^{\infty} \frac{1}{r^{1 / \gamma}(s)} \Delta s=\infty
$$

and

$$
\int^{\infty} \frac{1}{r^{1 / \gamma}(s)} \Delta s<\infty
$$

When $p(t) \equiv 0$, equation (1.1) reduces to the following equation

$$
\left(r(t)\left(y^{\Delta}(t)\right)^{\gamma}\right)^{\Delta}+q(t) y^{\beta}(t-\delta)=0, \quad t \in \mathbb{T} .
$$

In the super linear case, when $\gamma=\beta \geq 1$, the oscillation of the solution of equation (1.1) was discussed by Saker in [15] under the condition (1.2).

We note that if $\mathbb{T}=\mathbb{R}$ we have $\sigma(t)=t, \mu(t)=0, f^{\Delta}(t)=f^{\prime}(t)$. Then equation (1.1) becomes

$$
\left(r(t)\left([y(t)+p(t) y(t-\tau)]^{\prime}\right)^{\gamma}\right)^{\prime}+q(t) y^{\beta}(t-\delta)=0 \quad t \in \mathbb{R} .
$$

If $\mathbb{T}=\mathbb{N}$, we have $\sigma(n)=n+1, \mu(n)=1 y^{\Delta}(n)=\Delta y(n)=y(n+1)-y(n)$, then equation (1.1) becomes

$$
\Delta\left(r(n)(\Delta[y(n)+p(n) y(n-\tau)])^{\gamma}\right)+q(n) y^{\beta}(n-\delta)=0 \quad n \in \mathbb{N} .
$$

If $\mathbb{T}=h \mathbb{N}, h>0$, we have $\sigma(t)=t+h, \mu(t)=h$, and $y^{\Delta}(t)=\Delta_{h}(t)=\frac{y(t+h)-y(t)}{h}$, then equation (1.1) becomes

$$
\Delta_{h}\left(r(t)\left(\Delta_{h}[y(t)+p(t) y(t-\tau)]\right)^{\gamma}\right)+q(t) y^{\beta}(t-\delta)=0 \quad t \in h \mathbb{N}
$$

If $\mathbb{T}=q^{\mathbb{N}}=\left\{t: t=q^{n}, n \in \mathbb{N}\right\} ;, q>1$, we have $\sigma(t)=q(t), \mu(t)=(q-1) t$ and $y^{\Delta}=$ $\Delta_{q} y(t)=\frac{y(q t)-y(t)}{h}$, then equation (1.1) becomes

$$
\Delta_{q}\left(r(t)\left(\Delta_{q}[y(t)+p(t) y(t-\tau)]\right)^{\gamma}\right)+q(t) y^{\beta}(t-\delta)=0 \quad t \in q^{\mathbb{N}} .
$$


If $\mathbb{T}=\mathbb{N}^{2}=\left\{t^{2}: t \in \mathbb{N}\right\}$, we have $\sigma(t)=(\sqrt{t}+1)^{2}$ and $\mu(t)=1+2 \sqrt{t}$ and $y^{\Delta}(t)=\Delta_{N} y(t)=$ $\frac{y\left((\sqrt{t}+1)^{2}-y(t)\right)}{1+2 \sqrt{t}}$, then equation (1.1) becomes

$$
\Delta_{N}\left(r(t)\left(\Delta_{N}[y(t)+p(t) y(t-\tau)]\right)^{\gamma}\right)+q(t) y^{\beta}(t-\delta)=0 \quad t \in \mathbb{N}^{2} .
$$

If $\mathbb{T}=\left\{t_{n}: n \in \mathbb{N}\right\}$, where $\left\{t_{n}\right\}$ is the set of harmonic numbers defined by $t_{0}=0, t_{n}=$ $\sum_{k=1}^{n} \frac{1}{k}, n \in \mathbb{N}_{0}$, we have $\sigma\left(t_{n}\right)=t_{n+1}, \mu\left(t_{n}\right)=\frac{1}{n+1}$ and $y^{\Delta}(t)=\Delta t_{n} y\left(t_{n}\right)=(n+1) \Delta y\left(t_{n}\right)$, then equation (1.1) becomes

$$
\Delta t_{n}\left(r\left(t_{n}\right)\left(\Delta t_{n}\left[y\left(t_{n}\right)+p\left(t_{n}\right) y\left(t_{n}-\tau\right)\right]\right)^{\gamma}\right)+q\left(t_{n}\right) y^{\beta}\left(t_{n}-\delta\right)=0 \quad t \in \mathbb{T} .
$$

The paper is organized as follows: In the Section 2, we present some oscillation criteria of solutions of equation (1.1) when (1.2) holds. When (1.3) holds, we also establish some conditions which are sufficient for solutions of equation (1.1) to be oscillatory or converges to zero. Our results include those of Jinfa [13] and I.Kubiaczyk et.al.[14] when $\mathbb{T}=\mathbb{N}$ and $p(t)$ is identically zero. In Section 3, we present some examples to illustrate our main results.

\section{Oscillation criteria}

First, we consider the case, when condition (1.2) holds, and $\gamma \geq \beta \geq 1$. To prove our main result, we will use the following lemma which is called Keller's chain rule.

Lemma 2.1 ([3]). Let $f: \mathbb{R} \rightarrow \mathbb{R}$ be continuously differentiable and suppose $g: \mathbb{T} \rightarrow \mathbb{R}$ is delta differentiable. Then $f \circ g: \mathbb{T} \rightarrow \mathbb{R}$ is delta differentiable and the formula

$$
(f \circ g)^{\Delta}(t)=g^{\Delta}(t) \int_{0}^{1} f^{\prime}\left(g(t)+h \mu(t) g^{\Delta}(t)\right) d h
$$

holds.

Now we state and prove our main results.

Theorem 2.2. Assume that condition (1.2)holds. Furthermore assume that there exist positive rd-continuous delta differentiable functions $\alpha(t)$ and $\phi(t)$ such that for every $b \geq 1$ and $a$ positive number $M$,

$$
\lim _{t \rightarrow \infty} \sup \int_{t_{0}}^{t}\left[\alpha(s) \phi(s) Q(s)-\frac{K(s) C^{2}(s)}{4 \phi(s) \beta M^{\frac{\gamma-1}{\gamma}} \alpha(t)}\right] \Delta s=\infty,
$$

where

$$
\begin{aligned}
Q(s) & =q(s)(1-p(s-\delta))^{\beta}, C(s)=\frac{\phi(s)\left(\alpha^{\Delta}(s)\right)_{+}}{\alpha^{\sigma}}+\left(\phi^{\Delta}(s)\right)_{+} \\
K(t) & =(b \cdot(t-\delta))^{1-\beta}\left(\alpha^{\sigma}(t)\right)^{2} r^{\frac{1}{\gamma}}(t-\delta), \\
\left(\alpha^{\Delta}(t)\right)_{+} & =\max \left\{\alpha^{\Delta}(t), 0\right\} \text { and }\left(\phi^{\Delta}(t)\right)_{+}=\max \left\{\phi^{\Delta}(t), 0\right\},
\end{aligned}
$$

holds. Then every solution of equation (1.1) oscillates on $\left[t_{0}, \infty\right)_{\mathbb{T}}$. 
Proof. Suppose to the contrary that $y(t)$ is a nonoscillatory solution of equation (1.1). Let $t_{1} \geq$ $t_{0}$ be such that $y(t) \neq 0$ for all $t \geq t_{1}$. Without loss of generality, we may assume that $y(t)$ is an eventually positive solution of equation (1.1) with $y(t-\theta)>0$, where $\theta=\max \{\tau, \delta\}$ for all $t \geq t_{1}$ sufficiently large.

Set

$$
x(t)=y(t)+p(t) y(t-\tau) .
$$

From equation (1.1) and $\left(H_{2}\right)$ we have

$$
\left(r(t)\left(x^{\Delta}(t)\right)^{\gamma}\right)^{\Delta}=-q(t) y^{\beta}(t-\delta)<0 \quad \text { for all } t \in\left[t_{1}, \infty\right) \mathbb{T},
$$

and this implies that $r(t)\left(x^{\Delta}(t)\right)^{\gamma}$ is an eventually decreasing function. We first show that $r(t)\left(x^{\Delta}(t)\right)^{\gamma}$ is eventually nonnegative. If $r(t)\left(x^{\Delta}(t)\right)^{\gamma}<0$ for $t \geq t_{2} \geq t_{1}$, we have $r(t)\left(x^{\Delta}(t)\right)^{\gamma} \leq$ $r\left(t_{2}\right)\left(x^{\Delta}\left(t_{2}\right)\right)^{\gamma}=c<0$ for some constant $c$. Hence $x^{\Delta}(t) \leq\left(\frac{c}{r(t)}\right)^{\frac{1}{\gamma}}$. Now integrating from $t_{2}$ to $t$, we obtain

$$
x(t) \leq x\left(t_{2}\right)+c^{\frac{1}{\gamma}} \int_{t_{2}}^{t}\left(\frac{1}{r(s)}\right)^{\frac{1}{\gamma}} \Delta s
$$

which implied by $\left(H_{1}\right)$ that $x(t) \rightarrow-\infty$. This contradicts the fact that $x(t)>0$ for all $t \geq t_{1}$. Hence $r(t)\left(x^{\Delta}(t)\right)^{\gamma}$ is eventually nonnegative. Thus we see that there is some $t_{1}$ such that

$$
x(t)>0, x^{\Delta}(t) \geq 0, r(t)\left(x^{\Delta}(t)\right)^{\gamma}>0 \text {, and }\left(r(t)\left(x^{\Delta}(t)\right)^{\gamma}\right)^{\Delta} \geq 0 \text {, for } t \geq t_{1} .
$$

From (2.2) and (2.4), we have

$$
y(t)=x(t)-p(t) y(t-\tau) \geq(1-p(t)) x(t) .
$$

Then, for $t \geq t_{2}=t_{1}+\delta$, we have

$$
y(t-\delta) \geq(1-p(t-\delta)) x(t-\delta) .
$$

Using the last inequality in (2.3), we have

$$
\left(r(t)\left(x^{\Delta}(t)\right)^{\gamma}\right)^{\Delta}+q(t)(1-p(t-\delta))^{\beta} x^{\beta}(t-\delta) \leq 0 \text { for } t \in\left[t_{1}, \infty\right) \mathbb{\pi} .
$$

Define

$$
w(t)=\alpha(t) \frac{r(t)\left(x^{\Delta}(t)\right)^{\gamma}}{x^{\beta}(t-\delta)} \text { for } t \in\left[t_{2}, \infty\right) \mathbb{} .
$$

Then $w(t)>0$, and

$$
w^{\Delta}(t)=\left(r\left(x^{\Delta}\right)^{\gamma}\right)^{\sigma}\left[\frac{\alpha(t)}{x^{\beta}(t-\delta)}\right]^{\Delta}+\frac{\alpha(t)}{x^{\beta}(t-\delta)}\left(r(t)\left(x^{\Delta}(t)\right)^{\gamma}\right)^{\Delta}
$$




$$
\begin{aligned}
& =\frac{\alpha(t)}{x^{\beta}(t-\delta)}\left(r(t)\left(x^{\Delta}(t)\right)^{\gamma}\right)^{\Delta}+\left(r(t)\left(x^{\Delta}(t)\right)^{\gamma}\right)^{\sigma}\left[\frac{x^{\beta}(t-\delta) \alpha^{\Delta}(t)-\alpha(t)\left(x^{\beta}(t-\delta)\right)^{\Delta}}{x^{\beta}(t-\delta) x^{\beta}(\sigma(t)-\delta)}\right] \\
& \leq-\alpha(t) Q(t)+\frac{\left(\alpha^{\Delta}(t)\right)_{+}}{\alpha^{\sigma}(t)} w^{\sigma}(t)-\frac{\alpha(t)\left(r(t)\left(x^{\Delta}(t)\right)^{\gamma}\right)^{\sigma}\left(x^{\beta}(t-\delta)\right)^{\Delta}}{x^{\beta}(t-\delta) x^{\beta}(\sigma(t)-\delta)}
\end{aligned}
$$

The Keller's chain rule yields that

$$
\begin{aligned}
\left(x^{\beta}(t)\right)^{\Delta} & =\beta \int_{0}^{1}\left[h x^{\sigma}+(1-h) x\right]^{\beta-1} d h x^{\Delta}(t) \\
& \geq \beta(x(t))^{\beta-1} x^{\Delta}(t) .
\end{aligned}
$$

Then, for $t \in\left[t_{2}, \infty\right)_{\mathbb{T}}$ sufficiently large, we have

$$
\left(x^{\beta}(t-\delta)\right)^{\Delta}=\beta\left(x^{\beta-1}(t-\delta)\right)\left(x^{\Delta}(t-\delta)\right) .
$$

Also from (2.4) we have for $t \geq t_{2}$,

$$
r(t-\delta)\left(\left(x^{\Delta}(t-\delta)\right)^{\gamma}\right) \geq r(\sigma(t)-\delta)\left(x^{\Delta}(\sigma(t)-\delta)\right)^{\gamma} \geq\left(r(t)\left(x^{\Delta}\right)^{\gamma}(t)\right)^{\sigma},
$$

or

$$
x^{\Delta}(t-\delta) \geq \frac{\left(r^{\frac{1}{\gamma}}(t)\left(x^{\Delta}\right)\right)^{\sigma}}{r^{\frac{1}{\gamma}}(t)(t-\delta)} .
$$

Substitute the last inequality in (2.6), we obtain

$$
w^{\Delta}(t) \leq-\alpha(t) Q(t)+\frac{\left(\alpha^{\Delta}(t)\right)_{+}}{\alpha^{\sigma}(t)} w^{\sigma}(t)-\frac{\beta \alpha(t)}{r^{\frac{1}{\gamma}}(t-\delta)} \frac{\left(r^{\frac{1+\gamma}{\gamma}}(t)\left(x^{\Delta}(t)\right)^{\gamma+1}\right)^{\sigma}}{x^{\beta}(t-\delta) x^{\beta}(\sigma(t)-\delta)} x^{\beta-1}(t-\delta) .
$$

Since $x^{\Delta}(t) \geq 0$, we have $x(\sigma(t)-\delta) \geq x(t-\delta)$ and this implies that

$$
w^{\Delta}(t) \leq-\alpha(t) Q(t)+\frac{\left(\alpha^{\Delta}(t)\right)_{+}}{\alpha^{\sigma}(t)} w^{\sigma}(t)-\frac{\beta \alpha(t)}{r^{\frac{1}{\gamma}}(t-\delta)} \frac{\left(r^{\frac{1+\gamma}{\gamma}}(t)\left(x^{\Delta}(t)\right)^{\gamma+1}\right)^{\sigma}}{x^{2 \beta}(\sigma(t)-\delta)} x^{\beta-1}(t-\delta) .
$$

Form (2.5) and (2.7), we obtain

$$
w^{\Delta}(t) \leq-\alpha(t) Q(t)+\frac{\left(\alpha^{\Delta}(t)\right)_{+}}{\alpha^{\sigma}(t)} w^{\sigma}(t)-\frac{\beta \alpha(t) r^{\frac{1-\gamma}{\gamma}}(\sigma(t))}{r^{\frac{1}{\gamma}}(t-\delta)} \frac{\left(w^{\sigma}(t)\right)^{2}}{\left(\alpha^{\sigma}(t)\right)^{2}} \frac{1}{x^{1-\beta}(t-\delta)\left(x^{\Delta}(\sigma(t))^{\gamma-1}\right.} .
$$

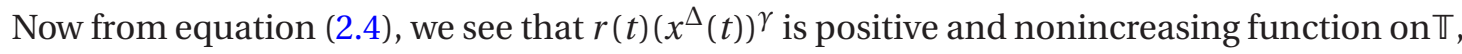
and therefore there exists a $t_{2} \in\left[t_{1}, \infty\right)_{\mathbb{T}}$ such that $r(t)\left(x^{\Delta}(t)\right)^{\gamma} \leq \frac{1}{M}$ for some positive constant $M$ and for $t \in\left[t_{2}, \infty\right) \pi$. Hence

$$
\frac{1}{x^{\Delta}(\sigma(t))^{\gamma-1}} \geq(M r(\sigma(t)))^{\frac{\gamma-1}{\gamma}}
$$


Further from (2.4) we have

$$
x(t)-x\left(t_{0}\right)=\int_{t_{0}}^{t} x^{\Delta}(s) \Delta s \leq x^{\Delta}\left(t_{0}\right)\left(t-t_{0}\right),
$$

and thus there exists a $T \in\left[t_{0}, \infty\right) \mathbb{\pi}$ and a suitable constant $b \geq 1$ such that

$$
x(t) \leq b t \text { for } t \in[T, \infty)_{\mathbb{T}} .
$$

Hence

$$
x^{1-\beta}(t-\delta) \leq b^{1-\beta}(t-\delta)^{1-\beta} \text { for } t \in\left[T_{1}, \infty\right) \mathbb{T},
$$

where $T_{1}=T+\delta$. Now substitute (2.9)and (2.10) in (2.8), we have

$$
w^{\Delta}(t) \leq-\alpha(t) Q(t)+\frac{\left(\alpha^{\Delta}(t)\right)_{+}}{\alpha^{\sigma}(s)} w^{\sigma}(t)-\frac{\beta M^{\frac{\gamma-1}{\gamma}} \alpha(t)}{(b \cdot(t-\delta))^{1-\beta}\left(\alpha^{\sigma}(t)\right)^{2} r^{\frac{1}{\gamma}}(t-\delta)}\left(w^{\sigma}(t)\right)^{2} .
$$

Multiplying (2.11) by $\phi(s)$ and integrating from $t_{2}$ to $t,\left(t \geq t_{2}\right)$, we have

$$
\begin{aligned}
\int_{t_{2}}^{t} \phi(s) \alpha(s) Q(s) \Delta s \leq & -\int_{t_{2}}^{t} \phi(s) w^{\Delta}(s) \Delta s+\int_{t_{2}}^{t} \phi(s) \frac{\left(\alpha^{\Delta}(s)\right)_{+}}{\alpha^{\sigma}(s)} w^{\sigma}(s) \Delta s \\
& -\int_{t_{2}}^{t} \phi(s) \frac{\beta M^{\frac{\gamma-1}{\gamma}} \alpha(s)}{\left.(b \cdot(s-\delta))^{1-\beta}\left(\alpha^{\sigma}(s)\right)^{2} r^{\frac{1}{\gamma}} s-\delta\right)}\left(w^{\sigma}(s)\right)^{2} \Delta s .
\end{aligned}
$$

Using integration by parts, we obtain

$$
\begin{aligned}
\int_{t_{2}}^{t} \phi(s) w^{\Delta}(s) \Delta s & =\left.\phi(t) w(t)\right|_{t_{2}} ^{t}-\int_{t_{2}}^{t}(\phi(s))^{\Delta} w^{\sigma}(s) \Delta s \\
& \leq-\phi\left(t_{2}\right) w\left(t_{2}\right)-\int_{t_{2}}^{t}\left(\phi^{\Delta}(s)\right)_{+} w^{\sigma}(s) \Delta s .
\end{aligned}
$$

From (2.12) and (2.13), we have

$$
\begin{aligned}
\int_{t_{2}}^{t} \phi(s) \alpha(s) Q(s) \Delta s \leq & w\left(t_{2}\right) \phi\left(t_{2}\right)+\int_{t_{2}}^{t}\left[\phi(s) \frac{\left(\alpha^{\Delta}(s)\right)_{+}}{\alpha^{\sigma}(s)}+\left(\phi^{\Delta}(s)\right)_{+}\right] w^{\sigma}(s) \Delta s \\
& -\int_{t_{2}}^{t} \phi(s) \frac{\beta M^{\frac{\gamma-1}{\gamma}} \alpha(s)}{(b \cdot(s-\delta))^{1-\beta}\left(\alpha^{\sigma}(s)\right)^{2} r^{\frac{1}{\gamma}}(s-\delta)} w^{2}(\sigma(s)) \Delta s,
\end{aligned}
$$

or

$$
\int_{t_{2}}^{t} \phi(s) \alpha(s) Q(s) \Delta s \leq w\left(t_{2}\right) \phi\left(t_{2}\right)+\int_{t_{2}}^{t}\left(\sqrt{\phi(s) \frac{\beta M^{\frac{\gamma-1}{r}} \alpha(s)}{K(s)}} w^{\sigma}(s)\right.
$$




$$
\left.-\frac{\sqrt{K(s)} C(s)}{2 \sqrt{\phi(s) \beta M^{\frac{\gamma-1}{\gamma}} \alpha(s)}}\right)^{2} \Delta s+\int_{t_{2}}^{t} \frac{K(s) C^{2}(s)}{4 \phi(s) \beta M^{\frac{\gamma-1}{\gamma}} \alpha(s)} \Delta s .
$$

Hence

$$
\lim _{t \rightarrow \infty} \int_{t_{2}}^{t}\left[\alpha(s) \phi(s) Q(s)-\frac{K(s) C^{2}(s)}{4 \phi(s) \beta M^{\frac{\gamma-1}{\gamma}} \alpha(s)}\right] \Delta s \leq w\left(t_{2}\right) \phi\left(t_{2}\right)<\infty,
$$

a contradiction to the condition (2.1). This completes the proof.

Remark 2.3. From Theorem 2.2, one can establish different sufficient conditions for the oscillation of equation (1.1) by different choices of $\alpha(t)$ and $\phi(t)$. For instance, if $\phi(t)=1$ for $t \geq t_{0}$ we have the following results.

Corollary 2.4. Assume condition (1.2) holds. Let $\alpha(t)$ be as defined in Theorem 2.2 such that for every $b \geq 1$ and a positive number $M$

$$
\lim _{t \rightarrow \infty} \sup \int_{t_{0}}^{t}\left[\alpha(s) q(s)(1-p(s-\delta))^{\beta}-\frac{K(s)\left(\frac{\left(\alpha^{\Delta}(s)\right)_{+}}{\alpha^{\sigma}(s)}\right)^{2}}{4 \beta M^{\frac{\gamma-1}{\gamma}} \alpha(s)}\right] \Delta s=\infty,
$$

where

$$
K(t)=b^{1-\beta}\left(\alpha^{\sigma}(t)\right)^{2} r^{\frac{1}{\gamma}}(t-\delta)(t-\delta)^{1-\beta},\left(\alpha^{\Delta}(t)\right)_{+}=\max \left\{\alpha^{\Delta}(t), 0\right\}
$$

Then every solution of equation (1.1) oscillates on $\left[t_{0}, \infty\right) \mathbb{4}$.

Corollary 2.5. Assume condition (1.2) holds. Let $\alpha(t)$ be as defined in Theorem 2.2 such that

$$
\lim _{t \rightarrow \infty} \sup \int_{t_{0}}^{t} \alpha(s) q(s)(1-p(s-\delta))^{\beta} \Delta s=\infty
$$

and

$$
\lim _{t \rightarrow \infty} \sup \int_{t_{0}}^{t} \frac{r^{\frac{1}{\gamma}}(s-\delta)(s-\delta)^{1-\beta}\left(\alpha^{\Delta}(s)\right)_{+}^{2}}{\alpha(s)} \Delta s<\infty .
$$

Then every solution of equation (1.1) oscillates on $\left[t_{0}, \infty\right)_{\mathbb{T}}$.

Remark 2.6. From Corollary 2.4, one can establish different sufficient conditions for the oscillation of equation (1.1) by different choices of $\alpha(t)$. For instance, if $\alpha(t)=t$ and $\alpha(t)=1$, for $t \geq t_{0}$, we have the following results respectively.

Corollary 2.7. Assume condition (1.2) holds. Furthermore assume that for every $b \geq 1$ and positive number $M$

$$
\lim _{t \rightarrow \infty} \sup \int_{t_{0}}^{t}\left[s q(s)(1-p(s-\delta))^{\beta}-\frac{b^{1-\beta} a^{\frac{1}{\gamma}}(s-\delta)(s-\delta)^{1-\beta}}{4 \beta M^{\frac{\gamma-1}{\gamma}} s}\right] \Delta s=\infty .
$$

Then every solution of equation (1.1) oscillates on $\left[t_{0}, \infty\right)_{\mathbb{T}}$. 
Corollary 2.8. Assume condition (1.2) holds. Let $\alpha(t)$ be as defined in Theorem 2.2 such that

$$
\lim _{t \rightarrow \infty} \sup \int_{t_{0}}^{t} q(s)(1-p(s-\delta))^{\beta} \Delta s=\infty
$$

Then every solution of equation (1.1) oscillates on $\left[t_{0}, \infty\right) \mathbb{\pi}$.

The following theorem gives Philos-type oscillation criteria for equation (1.1). First let us introduce a new class of functions $\mathscr{P}$ which will be extensively used in the sequel.

Let $\mathbb{D}_{0}=\left\{(t, s) \in \mathbb{T}^{2}: t>s \geq t_{0}\right\}$ and $\mathbb{D}=\left\{(t, s) \in \mathbb{T}^{2}: t>s \geq t_{0}\right\}$. The function $H \in C_{r d}(\mathbb{D}, \mathbb{R})$ is said to belongs to the class $\mathscr{P}$ if

(i) $H\left(t, t_{0}\right)=0, H(t, s)>0$ on $\mathbb{D}_{0}$

(ii) $\mathrm{H}$ has a continuous $\Delta$-partial derivative $H^{\Delta_{s}}(t, s)$ on $\mathbb{D}_{0}$ with respect to the second variable. ( $H$ is rd-continuous functions if $H$ is $r d$-continuous function in $t$ and $s$ ).

Theorem 2.9. Assume condition (1.2) holds. Furthermore, assume that there exists a positive $r d$-continuous delta differentiable functions $\alpha(t)$ and let $h, H: D \rightarrow R$ be rd-continuous functions such that $H$ belongs to the class $\mathscr{P}$ and for every $b \geq 1$ and a positive number $M$ with

$$
\lim _{t \rightarrow \infty} \sup \frac{1}{H\left(t, t_{0}\right)} \int_{t_{0}}^{t}\left[\alpha(s) H(t, s) Q(s)-\frac{K(s) C^{2}(t, s)}{4 \beta M^{\frac{\gamma-1}{\gamma}} H(t, s) \alpha(s)}\right] \Delta s=\infty,
$$

where $Q(t)=q(t)(1-p(t-\delta))^{\beta}, K(t)=(b \cdots(t \delta))^{1-\beta}\left(\alpha^{\sigma}(t)\right)^{2} r^{\frac{1}{\gamma}}(t-\delta)$, and $C(t, s)=\frac{H(t, s)\left(\alpha^{\Delta}(s)\right)}{\alpha^{\sigma}}+$ $H^{\Delta_{s}}(t, s)$. Then every solution of equation (1.1) oscillates on $\left[t_{0}, \infty\right) \mathbb{\pi}$.

Proof. Suppose to the contrary that $y(t)$ is a nonoscillatory solution of equation (1.1). Let $t_{1} \geq t_{0}$ be such that $y(t) \neq 0$ for all $t \geq t_{1}$. Without loss of generality, we may assume that $y$ is an eventually positive solution of equation (1.1) with $y(t-\theta)>0$ where $\theta=\max \{\tau, \delta\}$ for all $t \geq t_{1}$ sufficiently large. We proceed as in the proof of Theorem 2.2 to obtain that (2.4) holds for $t \geq t_{2}$. From (2.11), it follows that

$$
w^{\Delta}(t) \leq-\alpha(t) Q(t)+\frac{\left(\alpha^{\Delta}(t)\right)_{+}}{\alpha^{\sigma}(t)} w^{\sigma}(t)-\frac{\beta M^{\frac{\gamma-1}{\gamma}} \alpha(t)}{K(t)}\left(w^{\sigma}(t)\right)^{2} .
$$

Multiplying (2.20) by $H(t, s)$ on both sides and integrating from $t_{2}$ to $t$, we get

$$
\begin{aligned}
\int_{t_{2}}^{t} H(t, s) \alpha(s) Q(s) \Delta s \leq- & \int_{t_{2}}^{t} H(t, s) w^{\Delta}(s) \Delta s+\int_{t_{2}}^{t} \frac{H(t, s)\left(\alpha^{\Delta}(s)\right)_{+}}{\alpha^{\sigma}(s)} w^{\sigma}(s) \Delta s \\
& -\int_{t_{2}}^{t} H(t, s) \frac{\beta M^{\frac{\gamma-1}{\gamma}} \alpha(s)}{K(s)}\left(w^{\sigma}(s)\right)^{2} \Delta s .
\end{aligned}
$$


Using integration by parts we have

$$
\begin{aligned}
-\int_{t_{2}}^{t} H(t, s) w^{\Delta}(s) \Delta s & =-[H(t, s) w(s)]_{t_{2}}^{t}+\int_{t_{2}}^{t} H^{\Delta_{s}}(t, s) w^{\sigma}(s) \Delta s \\
& \leq H\left(t, t_{2}\right) w\left(t_{2}\right)+\int_{t_{2}}^{t} H^{\Delta_{s}}(t, s) w^{\sigma}(s) \Delta s .
\end{aligned}
$$

Substituting (2.22) in (2.21), we obtain

$$
\begin{aligned}
\int_{t_{2}}^{t} H(t, s) \alpha(s) Q(s) \Delta s \leq & w\left(t_{2}\right) H\left(t, t_{2}\right)+\int_{t_{2}}^{t}\left[\frac{H(t, s)\left(\alpha^{\Delta}(s)\right)_{+}}{\alpha^{\sigma}(s)}+\left(H(t, s)^{\Delta_{s}}\right)\right] w^{\sigma}(s) \Delta s \\
& -\int_{t_{2}}^{t} H(t, s) \frac{\beta M^{\frac{\gamma-1}{\gamma}} \alpha(s)}{K(s)}\left(w^{\sigma}(s)\right)^{2} \Delta s .
\end{aligned}
$$

This implies that

$$
\begin{aligned}
\int_{t_{2}}^{t} H(t, s) \alpha(s) Q(s) \Delta s \leq & w\left(t_{2}\right) H\left(t, t_{2}\right)+\int_{t_{2}}^{t} C(t, s) w^{\sigma}(s) \Delta s \\
& -\int_{t_{2}}^{t} H(t, s) \frac{\beta M^{\frac{\gamma-1}{\gamma}} \alpha(s)}{K(s)}\left(w^{\sigma}(s)\right)^{2} \Delta s .
\end{aligned}
$$

Then by completing the square, we have

$$
\int_{t_{2}}^{t} H(t, s) \alpha(s) Q(s) \Delta s \leq w\left(t_{2}\right) H\left(t, t_{2}\right)+\int_{t_{2}}^{t} \frac{K(s) C^{2}(t, s)}{4 \beta M^{\frac{\gamma-1}{\gamma}} H(t, s) \alpha(s)} \Delta s .
$$

Thus for all $t \geq t_{2}$, we have

$$
\int_{t_{2}}^{t}\left[H(t, s) \alpha(s) Q(s)-\frac{K(s) C^{2}(t, s)}{4 \beta M^{\frac{\gamma-1}{\gamma}} H(t, s) \alpha(s)}\right] \Delta s<H\left(t, t_{2}\right) w\left(t_{2}\right),
$$

and

$$
\lim _{t \rightarrow \infty} \sup \frac{1}{H\left(t, t_{2}\right)} \int_{t_{2}}^{t}\left[\alpha(s) H(t, s) Q(s)-\frac{K(s) C^{2}(t, s)}{4 \beta M^{\frac{\gamma-1}{\gamma}} H(t, s) \alpha(s)}\right] \Delta s<\infty,
$$

which contradicts to the condition (2.19). This completes the proof.

Corollary 2.10. Let assumption (2.19) in Theorem 2.9 be replaced by

$$
\lim _{t \rightarrow \infty} \sup \frac{1}{H\left(t, t_{0}\right)} \int_{t_{0}}^{t} \alpha(s) H(t, s) Q(s) \Delta s=\infty,
$$

and

$$
\lim _{t \rightarrow \infty} \sup \frac{1}{H\left(t, t_{0}\right)} \int_{t_{0}}^{t} \frac{K(s) C^{2}(t, s)}{H(t, s) \alpha(s)} \Delta s<\infty .
$$

Then every solution of equation (1.1) oscillates on $\left[t_{0}, \infty\right) \mathbb{\pi}$. 
Remark 2.11. With an appropriate choice of the functions $\mathrm{H}$ and $\mathrm{h}$, one can establish a number of oscillation criteria for equation (1.1) on different types of time scales.

For example, if $H(t, s)=(t-s)^{m}, t, s \in D$ with $m>1$ then it is clear that $\mathrm{H}$ belongs to the class $\mathscr{P}$ and $\left((t-s)^{m}\right)^{\Delta s} \leq-m(t-\sigma(s))^{m-1}$. We consider the following two cases

Case: 1 . If $\mu(t)=0$, then

$$
\left((t-s)^{m}\right)^{\Delta s}=-m(t-s)^{m-1} .
$$

Case: 2 . If $\mu(t) \neq 0$, then we have

$$
\begin{aligned}
\left((t-s)^{m}\right)^{\Delta s} & =\frac{1}{\mu(s)}\left[\left((t-\sigma(s))^{m}\right)-\left((t-s)^{m}\right)\right] \\
& \leq-m(t-s)^{m-1} .
\end{aligned}
$$

Then from (2.11) and (2.24), we have $\left((t-s)^{m}\right)^{\Delta s} \leq-m\left((t-\sigma(s))^{m-1}\right.$. From Theorem 2.9, we have the following Kamenev-type oscillation criteria for equation (1.1).

Corollary 2.12. Assume condition (1.2) holds. Furthermore assume that there exist a positive $r d$-continuous delta differentiable function $\alpha(t)$ and for every $b \geq 1$ and a positive number $M$ with

$$
\lim _{t \rightarrow \infty} \sup \frac{1}{t^{m}} \int_{t_{0}}^{t}\left[\alpha(s)(t, s)^{m} Q(s)-\frac{K(s) C^{2}(t, s)}{4 \beta M^{\frac{\gamma-1}{\gamma}}(t, s)^{m} \alpha(s)}\right] \Delta s \Delta s=\infty,
$$

where $m>1, C(t, s)=\frac{(t-s)^{m}\left(\alpha^{\Delta}(s)\right)_{+}}{\alpha^{\sigma}}-m\left((t-\sigma(s))^{m-1}, t \geq s \geq t \geq t_{0}\right.$. Then every solution of equation (1.1) oscillates on $\left[t_{0}, \infty\right) \pi$.

Also, one can use the factorial function $H(t, s)=(t-s)^{(k)}$ where $(t)^{(k)}=t(t-1)(t-$ 2)...( $t-k+1), t^{(0)}=1$ and establish new oscillation criteria for equation (1.1).

In this case

$$
\begin{aligned}
H^{\Delta s}(t-s)^{(k)} & =\frac{(t-\sigma(s))^{k}-(t-s)^{k}}{\mu(s)} \\
& =\frac{-(t-s)^{k}-(t-\sigma(s))^{k}}{\mu(s)} \\
& \geq-k(t-s)^{k-1} .
\end{aligned}
$$

Next, we consider the case when (1.3) holds and $0<\beta<1, \gamma \geq 1$.

Theorem 2.13. Assume that (1.3) holds. Furthermore, assume that there exist positive functions $\alpha(t)$ and $\phi(t)$ such that (2.1) holds and

$$
\int_{t_{0}}^{\infty} \frac{1}{r^{\frac{1}{\gamma}}(s)}\left(\int_{t_{1}}^{s} q(u)(1-p(u-\delta))^{\beta} \Delta u\right)^{\frac{1}{\gamma}} \Delta s=\infty,
$$

for some $t_{0} \in \mathbb{T}$. Then every solution $x(t)$ of equation (1.1) is either oscillatory or tends to zero as $t \rightarrow \infty$ 
Proof. Suppose to the contrary that $y(t)$ is a nonoscillatory solution of equation (1.1). Let $t_{1} \geq$ $t_{0}$ be such that $y(t) \neq 0$ for all $t \geq t_{1}$. Without loss of generality, we may assume that $y(t)$ is an eventually positive solution of equation (1.1) with $y(t-\theta)>0$, where $\theta=\max \{\tau, \delta\}$ for all $t \geq t_{1}$ sufficiently large. Let $x(t)=y(t)+p(t) y(t-\tau)$. Then $x(t)>0$ for all $t \in\left[t_{0}, \infty\right) \mathbb{\pi}$. From equation (1.1), we have $\left(r(t)\left(x^{\Delta}(t)\right)^{\gamma}\right)^{\Delta} \leq 0$.

Hence $r(t)\left(x^{\Delta}(t)\right)^{\gamma}$ is either eventually nonnegative or eventually negative. Since $r(t)>0$ and $\gamma$ is a ratio of odd positive integers, we have either $x^{\Delta}(t) \geq 0$ or $x^{\Delta}(t)<0$ for all $t \geq t_{1} \geq t_{0}$.

In the case, where $x^{\Delta}(t)$ is eventually nonnegative, we may follow the inequality (2.6) as in the proof of Theorem 2.2 and one obtains

$$
w^{\Delta}(t) \leq-\alpha(t) Q(t)+\frac{\left(\alpha^{\Delta}(t)\right)_{+}}{\alpha^{\sigma}} w^{\sigma}(t)-\frac{\alpha(t)\left(r\left(x^{\Delta}\right)^{\gamma}\right)^{\sigma}\left(x^{\beta}(t-\delta)\right)^{\Delta}}{x^{\beta}(t-\delta) x^{\beta}(\sigma(t)-\delta)} .
$$

Since $\beta \leq 1$, the Keller's chain rule yields that

$$
\begin{aligned}
\left(x^{\beta}(t)\right)^{\Delta} & =\beta \int_{0}^{1}\left[h x^{\sigma}+(1-h) x\right]^{\beta-1} d h x^{\Delta}(t) \\
& \geq \beta x^{\beta-1}(\sigma(t)) x^{\Delta}(t) .
\end{aligned}
$$

Then, for $t \in\left[t_{2}, \infty\right)_{\mathbb{T}}$ sufficiently large, we have

$$
\left(x^{\beta}(t-\delta)\right)^{\Delta}=\beta\left(x^{\beta-1}(\sigma(t)-\delta)\right)\left(x^{\Delta}(t-\delta)\right) .
$$

Since the remaining part of the proof is similar to that of Theorem 2.2, we omit the details.

If $x^{\Delta}(t)$ is eventually negative, then $\lim _{t \rightarrow \infty} x(t)=a \geq 0$. We assert that $a=0$. If not, then $x^{\beta}(t-\delta) \rightarrow a^{\beta}>0$ as $t \rightarrow \infty$. Then there exists $t_{1} \in\left[t_{0}, \infty\right) \mathbb{1}$ such that $x^{\beta}(t-\delta) \geq a^{\beta}$. From equation (1.1), we have

$$
\left(r(t)\left(x^{\Delta}(t)\right)^{\gamma}\right)^{\Delta} \leq-q(t)(1-p(t-\delta))^{\beta} a^{\beta} .
$$

Now integrating the last inequality from $t_{1}$ to $t$, we have

$$
\begin{aligned}
r(t)\left(x^{\delta}(t)\right)^{\gamma} & \leq r(t)\left(x^{\delta}(t)\right)^{\gamma}-r\left(t_{1}\right)\left(x^{\Delta}\left(t_{1}\right)\right)^{\gamma} \\
& \leq-a^{\beta} \int_{t_{1}}^{t} q(s)(1-p(s-\delta))^{\beta} \Delta s,
\end{aligned}
$$

or

$$
x^{\Delta}(t) \leq-a^{\frac{\beta}{\gamma}}\left(\frac{1}{r(t)} \int_{t_{1}}^{t} q(s)(1-p(s-\delta))^{\beta} \Delta s\right)^{\frac{1}{\gamma}} .
$$

Again integrating from $t_{1}$ to $t$, we obtain

$$
x(t) \leq x\left(t_{1}\right)-a^{\frac{\beta}{\gamma}} \int_{t_{1}}^{t} \frac{1}{r^{\frac{1}{\gamma}}(s)}\left(\int_{t_{1}}^{s} q(u)(1-p(u-\delta))^{\beta} \Delta u\right)^{\frac{1}{\gamma}} \Delta s .
$$


Condition (2.25) implies that $x(t)$ is eventually negative, which is a contradiction. Therefore $\lim _{t \rightarrow \infty} x(t)=0$. Since $x(t)=y(t)+p(t) y(t-\delta)$, then $0<y(t) \leq x(t)$. This implies that $y(t) \rightarrow 0$ as $t \rightarrow \infty$. The proof is complete.

\section{Examples}

In this section, we give some examples to illustrate our main results. To obtain the condition for oscillation, we will use the following facts.

$$
\int_{t_{0}}^{\infty} \frac{\Delta s}{s^{v}}=\infty, \text { if } \quad 0 \leq v \leq 1
$$

and

$$
\int_{t_{0}}^{\infty} \frac{\Delta s}{s^{v}}<\infty \text { if } \quad v>1
$$

Example 3.1. Consider the following second order neutral delay dynamic equation

$$
\left(\left(\left(y(t)+\frac{1}{2} y(t-\tau)\right)^{\Delta}\right)^{3}\right)^{\Delta}+\frac{1}{t+1} y^{5 / 3}(t-\delta)=0, \quad t \in[1, \infty)_{\mathbb{T}},
$$

$\tau$ and $\delta$ are non-negative constants such that $t-\tau$ and $t-\delta \in \mathbb{T}$. In this example $\gamma=3, \beta=$ $5 / 3, r(t)=1, q(t)=\frac{1}{t}, p(t)=\frac{1}{2}$. It is easy to see that assumptions $\left(H_{1}\right)-\left(H_{4}\right)$ hold. Choose $\phi(s)=1$ and $\alpha(s)=1$, then from Corollary 2.8 we have

$$
\underset{t \rightarrow \infty}{\limsup } \int_{t_{0}}^{t} q(s)(1-p(s-\delta))^{5 / 3} \Delta s=\limsup _{t \rightarrow \infty} \frac{1}{2^{5 / 3}} \int_{t_{0}}^{t} \frac{1}{s+1} \Delta s=\infty .
$$

Hence every solution of equation (3.1) is oscillatory.

Example 3.2. Consider the following second order neutral delay dynamic equation

$$
\left(\left(\left(y(t)+\frac{1}{2} y(t-\tau)\right)^{\Delta}\right)^{5}\right)^{\Delta}+\frac{1}{t^{2}} y^{5 / 3}(t-\delta)=0, \quad t \in[1, \infty)_{\mathbb{T}},
$$

$\tau$ and $\delta$ are non-negative constants such that $t-\tau$ and $t-\delta \in \mathbb{T}$. In this example $\gamma=5, \beta=$ $5 / 3, r(t)=1, q(t)=\frac{1}{t}, p(t)=\frac{1}{2}$. It is easy to see that assumptions $\left(H_{1}\right)-\left(H_{4}\right)$ hold. Choose $\phi(s)=1$ and $\alpha(s)=s$, then from Corollary 2.7 we have

$$
\begin{aligned}
\lim _{t \rightarrow \infty} \sup \int_{t_{0}}^{t}[ & \left.s q(s)(1-p(s-\delta))^{\beta}-\frac{b^{1-\beta} r^{\frac{1}{\gamma}}(s-\delta)(s-\delta)^{1-\beta}}{4 \beta M^{\frac{\gamma-1}{\gamma}} s}\right] \Delta s \\
= & \lim _{t \rightarrow \infty} \sup \int_{t_{0}}^{t}\left[\frac{1}{2^{5 / 3}} \frac{1}{s}-\frac{3}{20 b^{2 / 3} M^{4 / 5}} \frac{1}{s(s-\delta)^{2 / 3}}\right] \Delta s \\
= & \infty .
\end{aligned}
$$

Hence every solution of equation (3.2) is oscillatory. 
Example 3.3. Consider the following second order neutral delay dynamic equation

$$
\left[y(t)+\frac{1}{t+\delta} y(t-\tau)\right]^{\Delta \Delta}+\frac{\lambda}{t^{2}} y(t-\delta)=0 \quad t \in[1, \infty) \mathbb{\pi},
$$

$\tau$ and $\delta$ are non-negative constants such that $t-\tau$ and $t-\delta \in \mathbb{T}$ and $\lambda>0$ is a constant. For this equation, $\beta=\gamma=1, r(t)=1, f(t, u)=q(t) u, q(t)=\frac{\lambda}{t^{2}}, p(t)=\frac{1}{t+\delta}$ i.e., $p(t-\delta)=\frac{1}{t}$. It is easy to see that assumptions $\left(H_{1}\right)-\left(H_{4}\right)$ hold. Choose $\phi(s)=1$ and $\alpha(s)=s$, then from Corollary 2.7 we have

$$
\begin{aligned}
\lim _{t \rightarrow \infty} \sup \int_{t_{0}}^{t}[s q(s) & \left.(1-p(s-\delta))^{\beta}-\frac{b^{1-\beta} r^{\frac{1}{\gamma}}(s-\delta)(s-\delta)^{1-\beta}}{4 \beta M^{\frac{\gamma-1}{\gamma}} s}\right] \Delta s=\infty \\
& =\lim _{t \rightarrow \infty} \sup \int_{t_{0}}^{t}\left[\frac{\lambda}{s}\left(1-\frac{1}{s}\right)-\frac{1}{4 s}\right] \Delta s \\
& =\lim _{t \rightarrow \infty} \sup \int_{t_{0}}^{t}\left[\frac{\lambda}{s}-\frac{\lambda}{s^{2}}-\frac{1}{4 s}\right] \Delta s \\
& =\lim _{t \rightarrow \infty} \sup \int_{t_{0}}^{t}\left[\frac{4 \lambda-1}{4 s}-\frac{\lambda}{s^{2}}\right] \Delta s \\
& =\infty, \text { if } \lambda>\frac{1}{4} .
\end{aligned}
$$

Hence every solution of equation (3.3) oscillates if $\lambda>\frac{1}{4}$.

\section{References}

[1] R. P. Agarwal, M. Bohner and A. Peterson, Dynamic equations on time scales: A survey. J. Comp. Appl .Math., Special issue on dynamic equations on time scales, edited by R.P.Agarwal, M.Bohner and D.O'Regan (Preprint in Ulmer Seminare 5), 141 (2002), 1-26,.

[2] D. K. Anderson and A. Zafer, Nonlinear oscillation of second order dynamic equations on time scales, Appl. Math. Let., 22 (2009), 1591-1597.

[3] M. Bohner and A. Peterson, Dynamic Equations on Time Scales, An Introduction with Applications, Birkhuser, Boston, 2001.

[4] M. Bohner and A. Peterson, Advances in Dynamic Equations on Time Scales, Birkhuser, Boston, 2003.

[5] M.Bohner, S.H.Saker, Oscilltion criteria for pertubed nonlinear dynamic equations, Math. Comp. Model., 40 (2004), 249-260.

[6] M. Bohner, L. Erbe and A. Peterson, Oscillation for nonlinear second order dynamic equations on a time scales, J. Math. Anal. Appl., 301(2009), 491-507.

[7] L. Erbe, J. Baoguo and A. Peterson, Nonoscillation of second order sublinear dynamic equations on time scales, J. Comp. Appl. Math., 232 (2009), 594-599.

[8] L. Erbe, A. Peterson and S. H. Saker, Oscillation criteria for second order nonlinear delay dynamic equations, J. Math. Anal. Appl., 333 (2007), 505-522.

[9] S. R. Grace, R. Agarwal, M. Bohner and D. O'Regan, Oscillation of second order strongly superlinear and strongly sublinear dynamic equations, Comm. Nonl. Sci Num. Sim., 14 (2009), 3463-3471. 
[10] Z. Han, S. Sun and B. Shi, Oscillation criteria for a calss of second order Emden-Fowler delay dynamic equations on time scales, J. Math. Anal. Appl., 334 (2007), 847-858.

[11] S. Hilger, Analysis on measure chains - a unified approach to continuous and discrete analysis, Results Math., 8(1990), 18-56.

[12] M. Huang and W. Feng, Oscillation for forced second order nonlinear dynamic equations on time scales, Elect. J. Diff. Eqn., 145 (2005), 1-8.

[13] C. Jinfa, Kamenev type oscillation criteria for delay difference equations, Acta Math. Sci., 27(2007), 574-580.

[14] I. Kubiczyk, S. H. Saker and J. Morchalo, Kamenev type oscillation criteria for sublinear delay difference equations, Indian J. Pure Appl. Math., 34(2003), 1273-1284.

[15] S. H. Saker, Oscillation of second order nonlinear neutral delay dynamic equations, J. Comp. Appl. Math., 187 (2006), 123-141.

[16] S. H. Saker, D. O’Regan and R. P. Agarwal, Oscillation theorems for second order nonlinear neutral delay dynamic equations on time scales, Acta. Math. Sinica, English Series, 24(2007), 1409-1432.

[17] Y. Sahiner, Oscillation of second order delay differential equations on time scales, Nonlinear Analysis: theory, methods and applications, 63(2005), 173-1080.

[18] H. Wu, R. K. Zhuang, and R. M. Mathsen, Oscillation criteria for second-order nonlinear neutral variable delay dynamic equations, Appl. Math. Comp., 178(2006), 321-331.

[19] B. G. Zhang and Z. Shanliang, Oscillation of second order nonlinear delay dynamic equations on time scales, Comp. Math. Appl., 49 (2005), 599-609.

Ramanujan Institute for Advanced Study in Mathematics, University of Madras, Chennai, India.

E-mail: ethandapani@yahoo.co.in

Department of Mathematics, Bharathidasan University, Tiruchirappalli, India

E-mail: piramanantham@yahoo.co.in 